\title{
Prognostic biomarkers in stage IV non-small cell lung cancer (NSCLC): neutrophil to lymphocyte ratio (NLR), lymphocyte to monocyte ratio (LMR), platelet to lymphocyte ratio (PLR) and advanced lung cancer inflammation index (ALI)
}

\author{
Hiren Mandaliya ${ }^{1,2}$, Mark Jones ${ }^{3}$, Christopher Oldmeadow ${ }^{2,3}$, Ina IC Nordman ${ }^{1,2}$ \\ ${ }^{1}$ Medical Oncology, Calvary Mater Newcastle, Waratah, NSW, Australia; ${ }^{2}$ School of Medicine and Public Health, University of Newcastle, Australia; \\ ${ }^{3}$ Hunter Medical Research Institute, Newcastle, NSW, Australia \\ Contributions: (I) Conception and design: H Mandaliya, II Nordman; (II) Administrative support: H Mandaliya, II Nordman; (III) Provision of study \\ materials or patients: None; (IV) Collection and assembly of data: H Mandaliya, II Nordman; (V) Data analysis and interpretation: All authors; (VI) \\ Manuscript writing: All authors; (VII) Final approval of manuscript: All authors. \\ Correspondence to: Dr. Hiren Mandaliya. Calvary Mater Newcastle, Edith Street, Waratah, NSW, Australia. Email: hammandalia@gmail.com.
}

\begin{abstract}
Background: Currently, there is no single validated biomarker which can prognosticate survival in patients with stage IV non-small cell lung cancer (NSCLC). This study examines the prognostic significance of four biomarkers: neutrophil to lymphocyte ratio (NLR), lymphocyte to monocyte ratio (LMR), platelet to lymphocyte ratio (PLR) and advanced lung cancer inflammation index (ALI) in patients with stage IV NSCLC.

Methods: This study aimed to establish the relationship between NLR, LMR, PLR, ALI and overall survival (OS) at baseline and post first cycle of treatment using Cox univariate PH models. We also studied these biomarkers in the elderly (age $\geq 70$ years). Clinical data was sourced from Calvary Mater Newcastle between 2010 and 2015.

Results: Baseline NLR, PLR, LMR and ALI showed strong association with OS. Five unit increase in NLR and PLR was associated with an $11 \%$ and $0.5 \%$ increase in the hazard of death respectively while 1 unit increase in ALI resulted in 4\% increase in hazard of death. Five unit increase in LMR was associated with a $50 \%$ reduction in hazard of death. Post-treatment NLR and low ALI correlated with shorter OS but no statistically significant relationship could be demonstrated for PLR nor LMR. Similar prognostic trends were noted for elderly.

Conclusions: High NLR, high PLR, low LMR and low ALI at baseline are significantly associated with poor OS. High NLR and low ALI are significantly associated with poor OS post treatment. Findings are similar regardless of age.
\end{abstract}

Keywords: Prognostic; biomarker; lung cancer

Submitted Jun 15, 2019. Accepted for publication Aug 29, 2019.

doi: 10.21037/tlcr.2019.11.16

View this article at: http://dx.doi.org/10.21037/tlcr.2019.11.16

\section{Introduction}

Lung cancer is the leading cause of cancer death in the world and the second most common cancer diagnosed in both men and women $(1,2)$. Despite major advances in treatment the prognosis of patients with non-small cell lung cancer (NSCLC) is still poor. It is difficult to predict outcomes in individual patients. Therefore, there is a need for biomarkers which can predict treatment outcomes and so help to identify patients most likely to benefit from treatment. Factors such as extent of the 
disease, performance status and weight loss can be useful in predicting treatment benefits and prognosis in patients with lung cancer (3).

There have been extensive investigations carried out to find the best laboratory markers as a prognostic and predictive role in different cancer settings including NSCLC. The markers of systemic inflammatory response (SIR) such as plasma C-reactive protein (CRP), hypoalbuminemia, absolute white cell counts (WBC) or its components have been shown to have an important role in cancer development and progression (4,5). Moreover, the Glasgow prognostic score (based on serum CRP and albumin) was shown to be of prognostic value in predicting outcome $(\mathrm{HR}=1.70 ; 95 \% \mathrm{CI}: 1.23-2.35, \mathrm{P}=0.001)$ in inoperable stage II and IV NSCLC (6). Although, SIR has a predictive role in survival of incurable NSCLC patients receiving chemotherapy, the evidence for the use of these biomarkers [neutrophil to lymphocyte ratio (NLR), lymphocyte to monocyte ratio (LMR), platelet to lymphocyte ratio (PLR) and advanced lung cancer inflammation index (ALI)] as a predictor of outcome in patients with NSCLC at baseline and after receiving chemotherapy is limited as is the evidence of their relevance in the elderly.

NLR, LMR and PLR are the markers of SIR. Gu et al. in a study of 3,656 patients showed NLR to be potentially a useful biomarker to predict the poor prognosis for NSCLC (7). Lin et al. in a study of 370 patients showed LMR has the potential to be regarded as an independent prognostic factor for survival in previously untreated metastatic NSCLC (8). Similarly, Gu et al. in a study of 3,430 patients showed PLR to be associated with poor survival in advanced NSCLC (9). High NLR, low LMR and high PLR pretreatment predict shorter survival (7-9). ALI is an index derived from BMI (body mass index), albumin and NLR to quantify systemic inflammation (10). There is a growing interest to the potential prognostic and predictive role ALI may serve in a number of cancers including NSCLC. Ozyurek et al. in a study of 112 patients showed ALI to be an index of inflammation (11). NLR, LMR, PLR and ALI are easily measured and reproducible tests. The role of ALI at diagnosis is increasingly explored as an independent factor of survival in stage IV NSCLC. The variation in all these biomarkers post-treatment and their association with survival is not well studied.

Evolution of various predictive biomarkers have improved the therapy for patients with NSCLC with significant clinical benefits. However, there are no biomarkers which have emerged to help guide therapy in the elderly patients. The treatment in this cohort is often challenging as the elderly are likely to have more comorbidities, experience more drug interactions due to polypharmacy and are potentially more likely to experience toxicities from cytotoxics (12). As a result, this may result in under treatment (13). However, as the population ages, the number of patients aged $>70$ years in oncology clinics is steadily increasing. Hence, it is essential to identify biomarkers to assist with providing a maximum benefit from active treatment at a minimum risk of toxicities.

In this study, we evaluated the predictive and the prognostic role of the four biomarkers (NLR, LMR, PLR and ALI) of SIR on overall survival (OS) at the time of diagnosis and post first-cycle chemotherapy in patients with metastatic NSCLC in all age groups as well as the $\geq 70$ elderly. We also assessed which biomarker was most specific.

\section{Methods}

A retrospective descriptive study was conducted in patients with stage IV NSCLC actively treated at Calvary Mater Newcastle, a tertiary cancer centre in Australia from 2010 to 2015 . Relevant clinical and laboratory data were collected from electronic medical records system. Data on demographic variables, clinicopathological characteristics, treatment pattern, time of death and last follow-up information were collected and summarised. NLR, LMR, PLR and ALI $(\mathrm{ALI}=\mathrm{BMI} \times \mathrm{Albumin} / \mathrm{NLR} ; \mathrm{BMI}=$ body mass index, NLR = neutrophil-to-lymphocyte ratio, albumin $=$ serum albumin $\mathrm{g} / \mathrm{dL}$ ) calculated at diagnosis and post first chemotherapy/targeted treatment. We dichotomised NLR, PLR, LMR and ALI at 5, 185, 4 and 18 respectively exploring survival above and below threshold values as per published literature (7-10). Estimates of Kaplan-Meier (KM) survival distribution for OS generated. Extended Cox regression used to derive OS hazard ratios of predictive variables. The project was approved by the Research Ethics Committee.

To determine if the same patterns applied across the age groups across groups and to gain insight into the most useful biomarker in the elderly, we carried out a subgroup analysis of two patient groups $<70$ and $\geq 70$ years. Data were collected on patient demographics, clinico-pathological characteristics and survival.

\section{Response and predictor variables}

The baseline OS time was calculated as the difference 
Table 1 Patients demographic

\begin{tabular}{|c|c|}
\hline Characteristic & $\mathrm{N}=279$ \\
\hline Median age at diagnosis & 65 [29-85] \\
\hline Age $<70$ & $61[29-70]$ \\
\hline Age $\geq 70$ & 75 [70-85] \\
\hline \multicolumn{2}{|l|}{ Age group, n (\%) } \\
\hline$\geq 70$ & 78 [28] \\
\hline$<70$ & $201[72]$ \\
\hline \multicolumn{2}{|l|}{ Sex, n (\%) } \\
\hline Male & $166[59]$ \\
\hline Age $<70$ & $115[59]$ \\
\hline Age $\geq 70$ & $51[61]$ \\
\hline Female & $113[41]$ \\
\hline Age $<70$ & $81[41]$ \\
\hline Age $\geq 70$ & 32 [39] \\
\hline \multicolumn{2}{|l|}{ EGFR mutation } \\
\hline $\mathrm{Y}$ & $24[9]$ \\
\hline $\mathrm{N}$ & $198[71]$ \\
\hline NA & 57 [20] \\
\hline \multicolumn{2}{|l|}{ ALK mutation } \\
\hline $\mathrm{Y}$ & $3[1]$ \\
\hline $\mathrm{N}$ & $219[78]$ \\
\hline NA & 57 [20] \\
\hline \multicolumn{2}{|l|}{ Histology } \\
\hline Adenocarcinoma & 199 [71.3] \\
\hline Squamous cell carcinoma & $51[18.3]$ \\
\hline Large cell carcinoma & 27 [10] \\
\hline Mucinous adenocarcinoma & $1[0.4]$ \\
\hline
\end{tabular}

between the treatment start date and either death or the last observation recorded. The time to disease progression was calculated as the difference between the first line treatment start date and the disease progression date (or last observation if disease progression was not present).

NLR, PLR, LMR and ALI were hypothesised as potential predictors of death that may have prognostic value. NLR was calculated as absolute neutrophil count divided by absolute lymphocyte count, PLR was calculated platelet count divided by absolute lymphocyte count.
ALI was calculated as the product of BMI and albumin divided by the NLR $(A L I=B M I \times$ Albumin/NLR; BMI = body mass index, NLR = neutrophil-to-lymphocyte ratio, albumin = serum albumin $\mathrm{g} / \mathrm{dL}$ ).

\section{Analyses}

We provide descriptive statistics of patient characteristics and KM estimates for OS. For the first cycle of treatment, we modelled survival using the time between the first treatment and the last observation date. For the second cycle of treatment we modelled survival using the time between the second cycle treatment date and the last observation date (reflecting the OS conditional that the patient survived the first line of treatment).

We modelled disease progression with cumulative incidence from a competing risks model to estimate the occurrence of disease progression while accounting for the possibility of death appropriately.

In addition to cumulative incidence, we provide $\mathrm{KM}$ estimates of time to the composite event of disease progression or death.

Finally, we provide estimates from Cox Proportional Hazards models of the hazard ratios and visualisation based on $\mathrm{KM}$ estimates for a set of variables that were of prognostic interest.

The work was subsequently extended to provide a subgroup ( $<70 v s . \geq 70$ years of age) analysis.

All our statistical analyses were programmed using $R$ version 3.3.1 and cross-checked in Stata 14.

\section{Results}

Table 1 shows patients demographics, subset of patients stratified by age $<70$ and $\geq 70$ years and their histological/ molecular subtypes. The original data contained 280 patients. One patient was dropped from the analysis due to lack of information on last observation, disease progression and death.

\section{Survival analysis}

\section{First and second line treatment}

Of the 279 patients in the first line treatment, $226(81 \%)$ died and the remainder (53 patients) were right censored. Of the 226 patients at second line treatment, 182 (81\%) died. The median OS time of 10.5 months (95\% CI: 8.93 to 11.73 ) was noted for patients who received first line of 
A
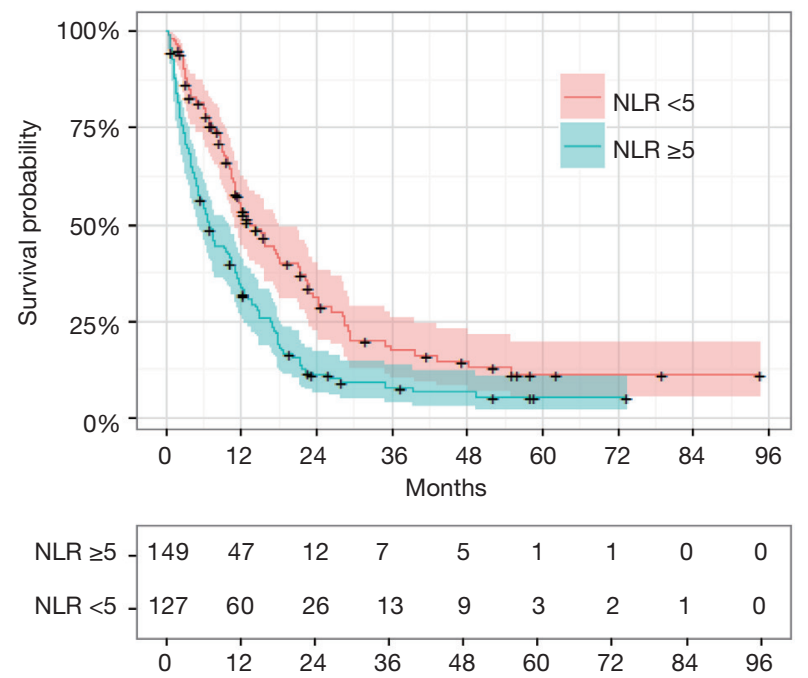

B

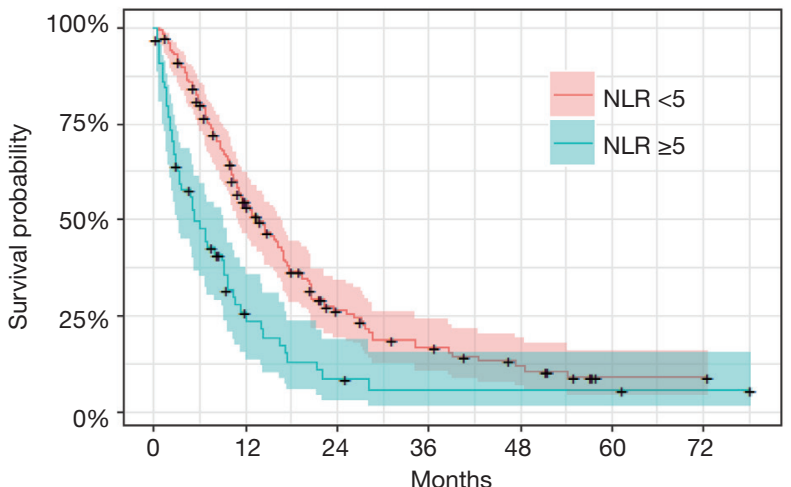

\begin{tabular}{|c|c|c|c|c|c|c|c|c|c|}
\hline$N L R \geq 5$ & 65 & 13 & 4 & 2 & 2 & 2 & 1 & 0 & 0 \\
\hline \multirow[t]{2}{*}{$N L R<5$} & 156 & 77 & 29 & 16 & 9 & 1 & 1 & 0 & 0 \\
\hline & 0 & 12 & 24 & 36 & 48 & 60 & 72 & 84 & 96 \\
\hline
\end{tabular}

Figure 1 KM overall survival by NLR at baseline (A) and the post second cycle treatment survival (B) using NLR $\geq 5$ and NLR $<5$.

treatment. Twelve months probability of survival was 0.42 (95\% CI: 0.36 to 0.48 ). For patients who received second line of treatment, their medical OS was 10.6 months $(95 \%$ CI: 9.5 to 12.67 ) and probability of survival at 12 months was 0.45 (95\% CI: 0.39 to 0.52 ). These findings were consistent for all patients and patients aged $\geq 70$ years.

\section{$N L R$}

There were 276 subjects with values for NLR at baseline. Of the 229 who received a second cycle of treatment, 221 had a NLR measurement at both the baseline and the second treatment cycle.

\section{Baseline}

Univariate CoxPH regression for OS suggested a hazard ratio for NLR at baseline of 1.022 (95\% CI: 1.015 to 1.028). Subgroup analyses identified the hazard ratio for NLR at baseline for the $<70$ years group was 1.021 (95\% CI: 1.014 to 1.029$)$ and the hazard ratio for the $\geq 70$ years group was 1.023 (95\% CI: 1.009 to 1.101), these differences were not statistically significant on the multiplicative scale ( $\mathrm{HR}=1.002 ; 95 \%$ CI: 0.986 to 1.018$)$.

\section{Second cycle}

There was evidence the effect of NLR at C2 changed over time (after adjusting for the baseline value of NLR2), the estimate for the hazard ratio for NLR at C2 was 1.095 (95\% CI: 1.038 to 1.122 ), and this reduced to 1.059 (95\% CI: 1.028 to 1.086$)$ at 6 months and was non-significant at
12 months, i.e., the prognostic utility of C2 NLR decreased over time. Subgroup analyses suggested a non-significant multiplicative difference 0.953 (95\% CI: 0.902 to 1.008 ) between the NLR C2 effects for the two age groups.

\section{NLR thresholds}

$\mathrm{KM}$ estimates of survival probabilities based on the dichotomised version of NLR at baseline (LHS) and NLR at the second treatment cycle with NLR $<5 v$. NLR $\geq 5$ are shown in Figure 1. For the first line treatment the median survival times were 13.37 months (95\% CI: 10.9-18.0) and 6.77 months (95\% CI: 5.1 to 10.2$)$ for NLR $<5$ vs. NLR $\geq 5$ respectively. For the second line treatment the median survival times were 13.67 months (95\% CI: 10.9 to 16.83 ) and 5.23 months (95\% CI: 3.0 to 9.07) for NLR $<5$ vs. NLR $\geq 5$ respectively.

\section{PLR}

Of the 279 subjects who received treatment at baseline, 276 had values for PLR and of the 229 that received a second cycle of treatment 223 had a PLR measurement at both baseline and the second cycle of treatment.

\section{Baseline}

Modelling OS from first treatment with a univariate CoxPH model gave an estimate for the hazard ratio for a unit increase in PLR at baseline of 1.001 (95\% CI: 1 to 1.001). The distribution of PLR at baseline was heavily skewed due to the presence of approximately 7 outliers with 
A

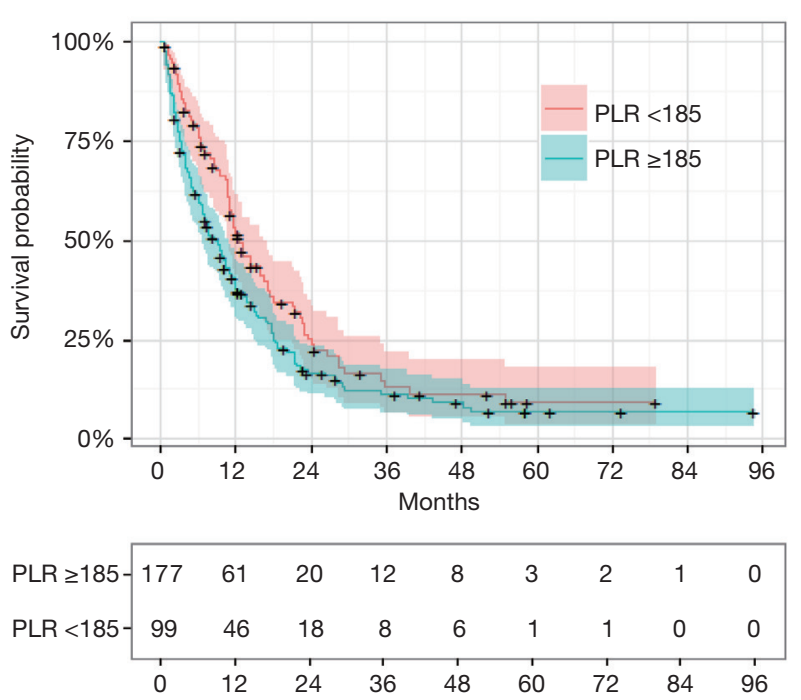

B

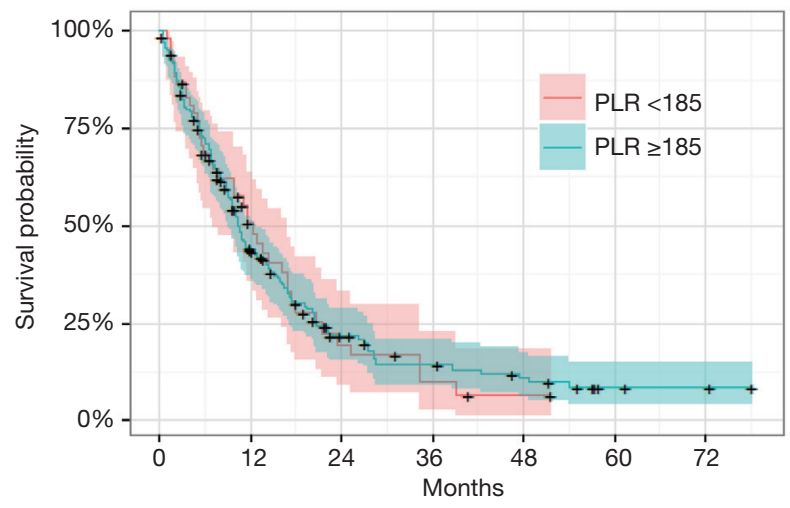

PLR $\geq 185-170=185-$\begin{tabular}{ccccccccc|}
170 & 70 & 26 & 15 & 10 & 3 & 2 & 0 & 0 \\
53 & 20 & 7 & 3 & 1 & 0 & 0 & 0 & 0 \\
0 & 12 & 24 & 36 & 48 & 60 & 72 & 84 & 96
\end{tabular}

Figure 2 KM overall survival by PLR at baseline (A) and the post second cycle treatment survival (B) using PLR $\geq 185$ and PLR $<185$.

values above 1,000 . However, the model results below were not sensitive to the removal of these points.

Subgroup analyses modelling OS including covariates for an interaction between PLR at baseline and age group membership and all lower order terms suggested a significant multiplicative difference of 1.002 (95\% CI: 1 to $1.004, \mathrm{P}<0.05$ ) between the PLR effects for the two groups.

\section{Second cycle}

There was a non-significant hazard ratio for PLR at the second cycle (HR $=0.9997,95 \%$ CI: 0.9991 to 1$)$. Subgroup analyses suggested a non-significant multiplicative difference of 1.001 (95\% CI: 0.999 to 1.002) between the PLR C2 rates for the two age groups.

\section{PLR thresholds}

Figure 2 provides the KM estimated survival probabilities based on a dichotomised version of PLR at baseline (LHS) and PLR at C2 with PLR $<185$ vs. PLR $\geq 185$. For the first line treatment the median survival times were 12.47 months (95\% CI: 10.87 to 16.5 ) and 8.77 months (95\% CI: 6.63 to 11.0$)$ for PLR $<185$ vs. PLR $\geq 185$ respectively. For the second cycle treatment the median survival times were 12.2 months (95\% CI: 7.13 to 16.8 ) and 10.3 months (95\% CI: 9.13 to 12.9$)$ for $\mathrm{PLR}<185$ vs. PLR $\geq 185$ respectively.

\section{$L M R$}

Of the $\mathrm{n}=279$ subjects receiving treatment at baseline, $\mathrm{n}=275$ had values for $\mathrm{LMR}$ and of the $\mathrm{n}=229$ that received a second cycle of treatment $n=217$ had LMR measurement both-pre-treatment and at the second cycle of treatment.

\section{Baseline}

A time dependent effect LMR at baseline on OS was observed. Initially the hazard ratio for LMR at baseline was 0.867 (95\% CI: 0.715 to 0.995 ) suggesting a unit increase in LMR at baseline was associated with a $13 \%$ reduction in risk. However, the hazard ratio was not significant at 6 months with a value of 0.924 (95\% CI: 0.792 to 1.031 ). This suggests that the prognostic utility of LMR decreases rapidly over time.

Subgroup analyses suggested a non-significant multiplicative difference of 0.981 (95\% CI: 0.757 to 1.273 ) between the LMR rates for the two age groups.

\section{Second cycle}

There was a non-significant hazard ratio for LMR at C2 of 0.873 (95\% CI: 0.741 to 1.028 ). Subgroup analyses suggested a non-significant multiplicative difference of 1.216 (95\% CI: 0.980 to 1.508 ) between the LMR rates for the two age groups.

\section{LMR thresholds}

Figure 3 provides the KM estimated survival probabilities based on a dichotomised version of LMR at baseline (LHS) and $\mathrm{LMR}$ at $\mathrm{C} 2$ with $\mathrm{LMR}<4$ vs. $\mathrm{LMR} \geq 4$. For the first line treatment the median survival times were 10.3 months (95\% 
A
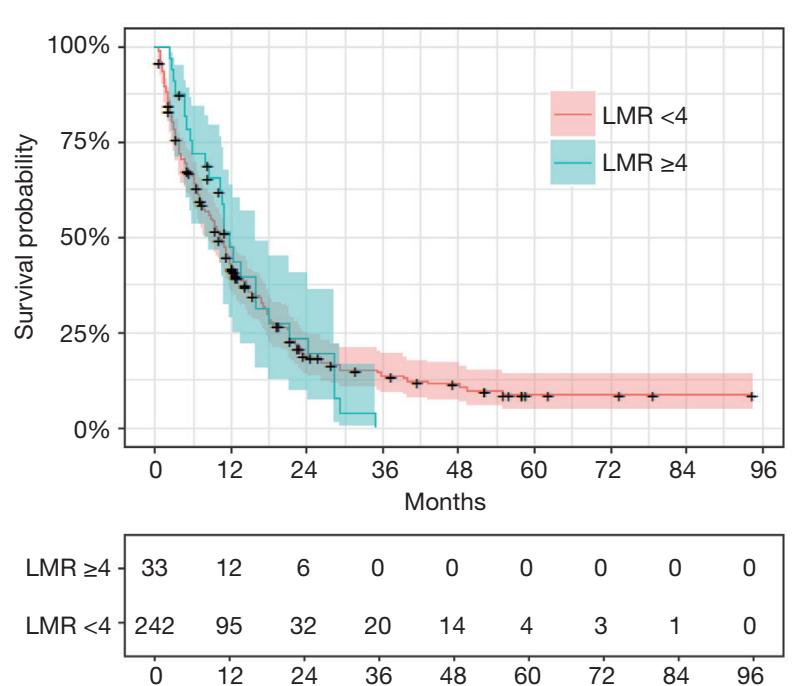

B

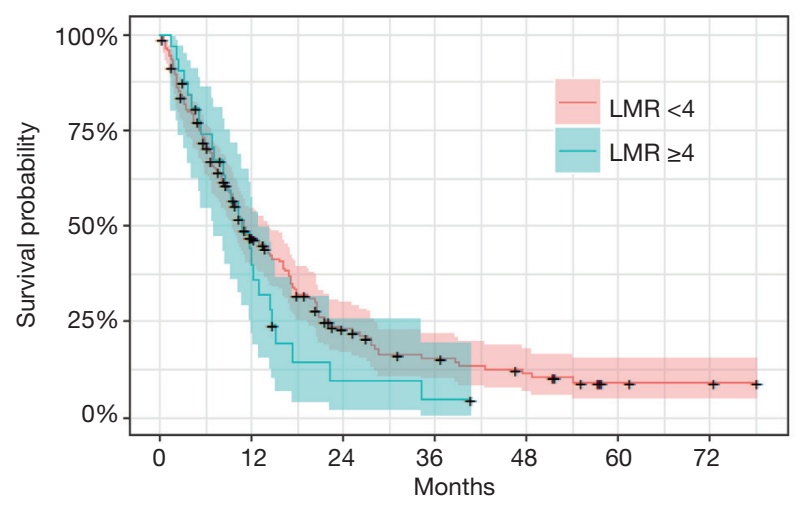

\begin{tabular}{|c|c|c|c|c|c|c|c|c|c|}
\hline$L M R \geq 4$ & 32 & 11 & 2 & 1 & 0 & 0 & 0 & 0 & 0 \\
\hline LMR $<4$ & 185 & 78 & 30 & 17 & 11 & 3 & 2 & 0 & 0 \\
\hline & 0 & 12 & 24 & 36 & 48 & 60 & 72 & 84 & 96 \\
\hline
\end{tabular}

Figure $3 \mathrm{KM}$ overall survival by LMR at baseline (A) and the post second cycle treatment survival (B) using LMR $\geq 4$ and LMR $<4$.

CI: 7.83 to 11.7$)$ and 11.9 months (95\% CI: 8.60 to 15.9 ) for $\mathrm{LMR}<4$ vs. LMR $\geq 4$ respectively. For the second cycle treatment the median survival times were 11.0 months $(95 \%$ CI: 9.53 to 14.3 ) and 10.8 months (95\% CI: 7.13 to 12.9 ) for $L M R<4$ vs. $L M R \geq 4$ respectively.

\section{$A L I$}

Of the $\mathrm{n}=279$ subjects receiving treatment at baseline, $\mathrm{n}=276$ had values for ALI and of the $\mathrm{n}=229$ that received a second round of treatment $n=189$ had ALI measurement at both baseline and the second cycle of treatment.

\section{Baseline}

A time-dependent effect was observed such that the hazard ratio for ALI at baseline was slowly increasing with time. Initially the hazard ratio for ALI was 0.957 (95\% CI: 0.943 to 0.972 ) suggesting a unit increase in ALI at baseline was associated with a $4 \%$ reduction in risk of mortality. At 6 months, the hazard ratio was 0.969 (95\% CI: 0.956 to 0.987 ) and at 12 months 0.982 (95\% CI: 0.971 to 0.994 ). Subgroup analyses suggested a non-significant multiplicative difference of 1.008 (95\% CI: 0.987 to 1.029 ) between the ALI rates for the two age groups.

\section{Second cycle}

Modelling second cycle treatment survival for ALI at the second treatment cycle gave a hazard ratio for ALI at the second treatment cycle of 1.001 (95\% CI: 0.999 to 1.005).
Subgroup analyses suggested a non-significant multiplicative difference of 1.003 (95\% CI: 0.9916 to 1.0142 ) between the ALI rates for the two age groups.

\section{ALI thresholds}

Figure 4 provides the KM estimated survival probabilities based on a dichotomised version of ALI at baseline and ALI at $\mathrm{C} 2$ with $\mathrm{ALI}<18$ vs. ALI $\geq 18$. For the first line treatment the median survival times were 6.23 months (95\% CI: $4.83-$ 9.27) and 14.70 months (95\% CI: 11.63-18.20) for ALI $<18$ vs. ALI $\geq 18$ respectively. For the second cycle treatment the median survival times were 5.23 months (95\% CI: $3.27-9.07)$ and 12.67 months (95\% CI: $10.47-15.13)$ for ALI $<18$ vs. ALI $\geq 18$ respectively.

\section{Summary of potential prognostic measures}

Table 2 shows a summary of the hazard ratios for OS from baseline and second cycle treatment for each of the nominated prognostic measures.

While NLR, PLR, LMR and ALI showed significant associations with survival from baseline at the 0.05 level, only NLR showed an association with survival from second line treatment.

\section{Discussion}

This comprehensive study demonstrated a significant association between pre-treatment (at baseline) NLR, PLR, 
A

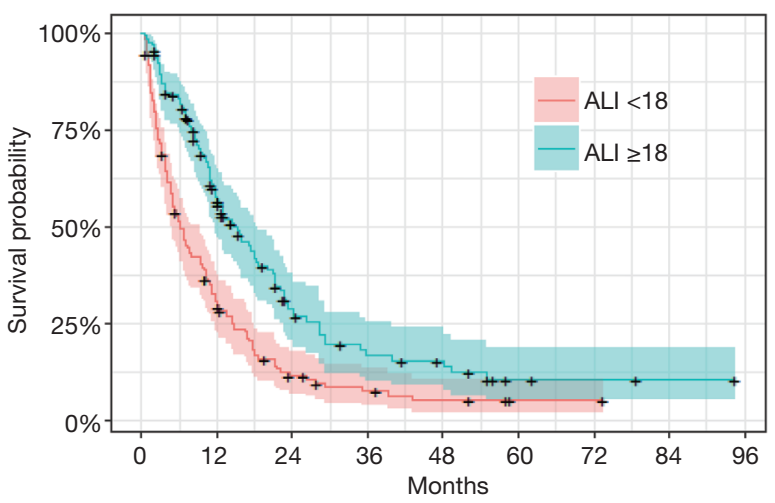

ALI $\geq 18-$\begin{tabular}{ccccccccc|}
132 & 66 & 25 & 13 & 10 & 3 & 2 & 1 & 0 \\
144 & 41 & 13 & 7 & 4 & 1 & 1 & 0 & 0 \\
0 & 12 & 24 & 36 & 48 & 60 & 72 & 84 & 96
\end{tabular}

B

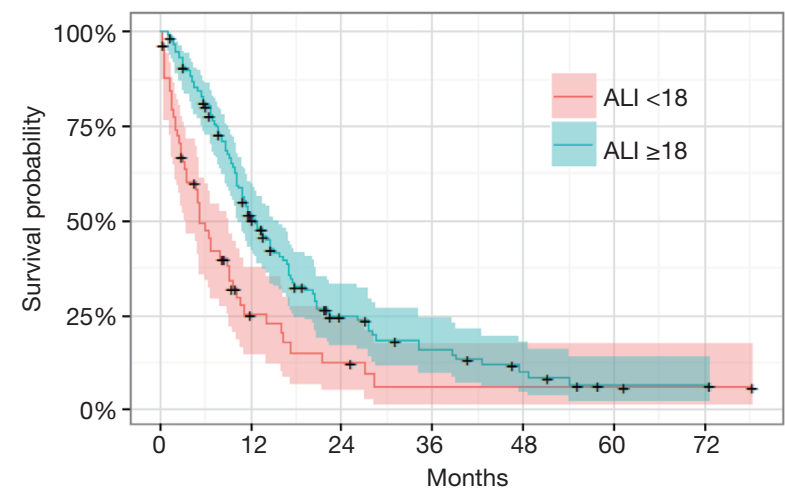

ALI $\geq 18-$\begin{tabular}{rrrrrrrrr|}
130 & 62 & 22 & 12 & 6 & 1 & 1 & 0 & 0 \\
\hline 59 & 11 & 5 & 2 & 2 & 2 & 1 & 0 & 0 \\
1 & 12 & 24 & 36 & 48 & 60 & 72 & 84 & 96
\end{tabular}

Figure $4 \mathrm{KM}$ overall survival by ALI at baseline (A) and the post second cycle treatment survival (B) using ALI $\geq 18$ and ALI $<18$.

Table 2 Estimates for hazard ratios for overall survival from first and second line treatment

\begin{tabular}{|c|c|c|c|c|}
\hline Group & Timepoint & Hazard ratio $(95 \% \mathrm{Cl})$ & $P$ value & C-stat \\
\hline NLR & Second line $(n=221)$ & 1.095 (1.038 to 1.122$)$ & $<0.001$ & 0.649 \\
\hline \multirow[t]{2}{*}{ PLR } & Baseline $(n=276)$ & 1.001 (1 to 1.001$)$ & $<0.001$ & 0.591 \\
\hline & Second line $(n=223)$ & 1 (0.9991 to 1$)$ & 0.23 & 0.593 \\
\hline LMR & Second line $(n=217)$ & 0.873 (0.741 to 1.028$)$ & 0.10 & 0.562 \\
\hline \multirow[t]{2}{*}{ ALI } & Baseline $(n=276)$ & 0.957 (0.940 to 0.981$)$ & $<0.001$ & 0.67 \\
\hline & Second line $(n=189)$ & $1.002(0.999$ to 1.004$)$ & 0.18 & 0.627 \\
\hline
\end{tabular}

LMR and ALI with OS. Post first-cycle treatment, only NLR and ALI showed association with OS as compared to PLR and LMR. A higher NLR at diagnosis is a negative prognostic factor for OS in stage IV NSCLC. Additionally, post-first cycle treatment, high NLR predicts poor OS in this group of patients. OS was shorter for high NLR and high PLR at baseline compared to low NLR and low PLR at baseline. OS was long for high LMR at baseline compared to low at baseline. An increase in NLR post firstcycle treatment correlated with a shorter OS. Pre-treatment (Baseline) levels of these prognostic biomarkers have shown similar prognostic utility as published in literature $(8,14,15)$. We have gone one step further and explored predictive role of this biomarkers post treatment.

There was also a strong relationship established between
ALI and survival at baseline. High pretreatment ALI predicted for a longer survival while low pretreatment ALI predicted for shorter survival. Role of ALI in this subgroup of lung cancer patients is consistent and similar to with published literature by Jafri et al. (10).

In a separate analysis, the importance of these markers was further explored in stage IV NSCLC patients aged $\geq 70$ years, as this group is heavily represented in the clinical practice. This was the first ever attempt to find the most suitable biomarker in this group of patients. The analysis confirmed a statistically significant association between NLR at baseline and $O S$ in patients aged $\geq 70$ years with stage IV NSCLC. Although, clinically there appeared to be a trend (pre and post treatment) for a better OS with low PLR, low LMR and high ALI, no statistically significant 
association could be demonstrated at baseline and post first cycle treatment with OS and PLR, LMR and ALI in this subgroup.

These four biomarkers, NLR, LMR, PLR and ALI represent a simple and an inexpensive tool for evaluation of systemic inflammation. By establishing their role which is available to most oncologists worldwide, there is a real possibility for these biomarkers to have clinical use in the future. These may allow for early stratification of patients for treatment and perhaps be used in clinics to establish the time needed for a change of treatment.

Although the biomarkers were derived prospectively, the present study is limited by being a single centre retrospective study. Therefore, further prospective validation is required to support the use of NLR, LMR, PLR and ALI to be used in prognostic and predictive models for stage IV NSCLC.

\section{Conclusions}

To the best of our knowledge, this is the first study examining the prognostic significance of all four markers of systemic inflammation (NLR, PLR, LMR and ALI) in patients with untreated and treated stage IV NSCLC.

For the first time, these biomarkers have also been explored in elderly (aged $\geq 70$ years) lung cancer patients for prognostic and predictive utility.

While NLR, PLR, LMR and ALI showed significant associations with OS prior to treatment, only NLR and ALI showed an association with OS after treatment. Similar utility of these biomarkers was noted among patients age $\geq 70$ in this group.

These simple biomarkers can be easily incorporated into routine clinical practice.

\section{Acknowledgments}

Hunter Cancer Research Alliance - Statistical support grant.

\section{Footnote}

Conflicts of Interest: The authors have no conflicts of interest to declare.

Ethical Statement: The authors are accountable for all aspects of the work in ensuring that questions related to the accuracy or integrity of any part of the work are appropriately investigated and resolved. The project was approved by the Research Ethics Committee.

\section{References}

1. Australian Institute of Health and Welfare. Cancer in Australia: Actual incidence data from 1982 to 2013 and mortality data from 1982 to 2014 with projections to 2017 . Asia Pac J Clin Oncol 2018;14:5-15.

2. Siegel RL, Miller KD, Jemal A. Cancer statistics, 2019. CA Cancer J Clin 2019;69:7-34.

3. Satoh H, Ishikawa H, Ohara G, et al. Long-term survivors after chemotherapy in advanced non-small cell lung cancer. Anticancer Res 2007;27:4457-60.

4. Balkwill F, Mantovani A. Inflammation and cancer: back to Virchow? Lancet 2001;357:539-45.

5. Coussens LM, Werb Z. Inflammation and cancer. Nature 2002;420:860-7.

6. Forrest LM, McMillan DC, McArdle CS, et al. Evaluation of cumulative prognostic scores based on the systemic inflammatory response in patients with inoperable non-small-cell lung cancer. Br J Cancer 2003;89:1028-30.

7. Gu XB, Tian T, Tian XJ, et al. Prognostic significance of neutrophil-to-lymphocyte ratio in non-small cell lung cancer: a meta-analysis. Sci Rep 2015;5:12493.

8. Lin GN, Peng JW, Xiao JJ, et al. Prognostic impact of circulating monocytes and lymphocyte-to-monocyte ratio on previously untreated metastatic non-small cell lung cancer patients receiving platinum-based doublet. Med Oncol 2014;31:70.

9. Gu X, Sun S, Gao XS, et al. Prognostic value of platelet to lymphocyte ratio in non-small cell lung cancer: evidence from 3,430 patients. Sci Rep 2016;6:23893.

10. Jafri SH, Shi R, Mills G. Advance lung cancer inflammation index (ALI) at diagnosis is a prognostic marker in patients with metastatic non-small cell lung cancer (NSCLC): a retrospective review. BMC Cancer 2013;13:158.

11. Ozyurek BA, Ozdemirel TS, Ozden SB, et al. Does advanced lung inflammation index (ALI) have prognostic significance in metastatic non-small cell lung cancer? Clin Respir J 2018;12:2013-9.

12. Repetto L. Greater risks of chemotherapy toxicity in elderly patients with cancer. J Support Oncol 2003;1:18-24.

13. Swaminathan D, Swaminathan V. Geriatric oncology: problems with under-treatment within this population. Cancer Biol Med 2015;12:275-83. 
14. Cedres S, Torrejon D, Martinez A, et al. Neutrophil to lymphocyte ratio (NLR) as an indicator of poor prognosis in stage IV non-small cell lung cancer. Clin Transl Oncol 2012;14:864-9.

Cite this article as: Mandaliya $\mathrm{H}$, Jones $\mathrm{M}$, Oldmeadow $\mathrm{C}$, Nordman II. Prognostic biomarkers in stage IV non-small cell lung cancer (NSCLC): neutrophil to lymphocyte ratio (NLR), lymphocyte to monocyte ratio (LMR), platelet to lymphocyte ratio (PLR) and advanced lung cancer inflammation index (ALI). Transl Lung Cancer Res 2019;8(6):886-894. doi: 10.21037/ tlcr.2019.11.16
15. Qiang G, Liang C, Xiao F, et al. Prognostic significance of platelet-to-lymphocyte ratio in non-small-cell lung cancer: a meta-analysis. Onco Targets Ther 2016;9:869-76. 